\title{
Agile Filterless Optical Networking
}

\author{
Christine Tremblay, Paul Littlewood, Michel P. Bélanger, Lena Wosinska and Jiajia Chen
}

\begin{abstract}
Filterless optical networks based on broadcast-and-select nodes equipped with coherent transceivers can be considered as very attractive solutions for cost-effective and flexible capacity allocation in terrestrial and submarine applications. In this paper, we present an overview of the research on filterless optical networking in the last 10 years.
\end{abstract}

Index Terms-Optical networks, filterless optical networks, coherent optical communication, gridless network architecture, active photonic switching, broadcast-and-select, routing and spectrum assignment (RSA), agile undersea optical networks.

\section{INTRODUCTION}

$\mathrm{I}$ $\mathrm{N}$ the face of relentless capacity growth, the filterless optical network concept was proposed initially as an attempt to minimize additional capital cost required for network agility without losing (or improving upon) the operational advantages of an agile network [1]. The proposed architecture eliminates or minimizes the active photonic reconfigurable component count in the optical line system, thereby reducing the installed first cost (IFC) of the network. Taking advantage of transmission technology breakthroughs such as electronic impairment compensation, tunable transmitters and coherent receivers, the concept was based on the premise that agility could now be achieved by wavelength tuning at the transmitter and wavelength discrimination at the receiver, using field proven processes similar to those in radio networks.

After ten years of research on the topic, what have we learned on filterless optical networking? First, agility using this broadcast-and select architecture can be realized but with some trade-offs. Here agility is understood as ease in reconfiguring the connectivity in the network. Secondly, this architecture is considered as a promising approach for software defined network (SDN) control techniques. Thirdly, the inherently passive gridless architecture of filterless networks makes them inherently suitable for elastic optical networking methods. Filterless networks are now starting to be explored and sometimes deployed by network operators as appealing

Manuscript submitted on March 11, 2017. This work was supported by the Natural Sciences and Engineering Research Council (NSERC) of Canada under grants CRDPJ 435362-12 and CRDPJ 335465-05, as well as by Nortel Networks and Ciena Corporation.

C. Tremblay is with the Department of Electrical Engineering, École de technologie supérieure, 1100, rue Notre-Dame Ouest, Montréal, Québec, H3C 1K3, Canada (e-mail: christine.tremblay@etsmtl.ca).

P. Littlewood and M. P. Bélanger are with Ciena Corp., 3500 Carling Avenue, Nepean, ON, Canada, K2H 8E9 (e-mail: plittlew@ciena.com).

L. Wosinska and J. Chen are with KTH Royal Institute of Technology, Electrum 229, 16440 Kista, Sweden (e-mail: wosinska@kth.se). commercial solutions for certain network applications. In fact, filterless optical networks have been trialed and deployed in Europe since 2012 [2-4].

The paper is organized as follows. The concept and advantages of filterless networks are presented in Section II. Section III looks at the performance and design trade-offs for filterless networks. Solutions to overcome the challenges and potential issues with filterless networks are discussed in Section IV. Finally, Section V provides concluding remarks and potential avenues for future work.

\section{CONCEPTS AND ADVANTAGES}

The concept of filterless optical networks, first introduced in [1], leverages the breakthroughs of coherent transmission and electronic impairment compensation technologies to offer network agility and cost efficiency. Active switched (the method currently deployed in most networks) and filterless photonic networks are contrasted in Fig. 1. Instead of deploying reconfigurable optical add-drop multiplexers (ROADMs) based on wavelength selective switches (WSSs) at network nodes, as in conventional active photonic networks, filterless networks use passive optical splitters and combiners for fiber interconnections and agile edge nodes equipped with coherent transponders. The elimination of active switching and filtering components creates a broadcast and select architecture in which agility is provided at the edge terminals by tunable coherent transceivers. Coherent technology is fundamental to terminal and system agility by performing the selection of the desired wavelength at the drop site.

Filterless optical networks exhibit several advantages compared to active switching networks. The removal of active (WSS) elements translates into simplified optical line systems, lowering cost and footprint, reducing power consumption, improving robustness and mean time between failures (MTBF), and simplifying aspects of impairment-aware design. The gridless architecture of filterless networks makes them inherently suitable for elastic optical networking and gridless innovations such as dynamic spectrum allocation. Moreover the filterless design enables colorless node operation, as optical terminals are able to access all DWDM channels and send/receive wavelengths as specified. Therefore, the transition from fixed grid to gridless operation can be achieved at minimal cost. The fiber trees in filterless optical networks also intrinsically support multicast traffic. Other advantages include easier network planning, and simpler and faster connection establishment. The passive bypass and add-drop functionality at intermediate nodes are key enablers for multilayer networking, allowing the traffic from the Internet 


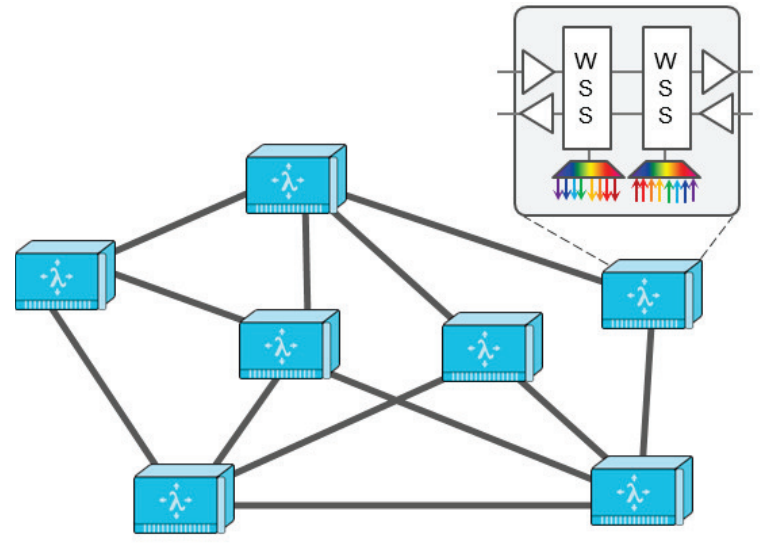

Active switching architecture

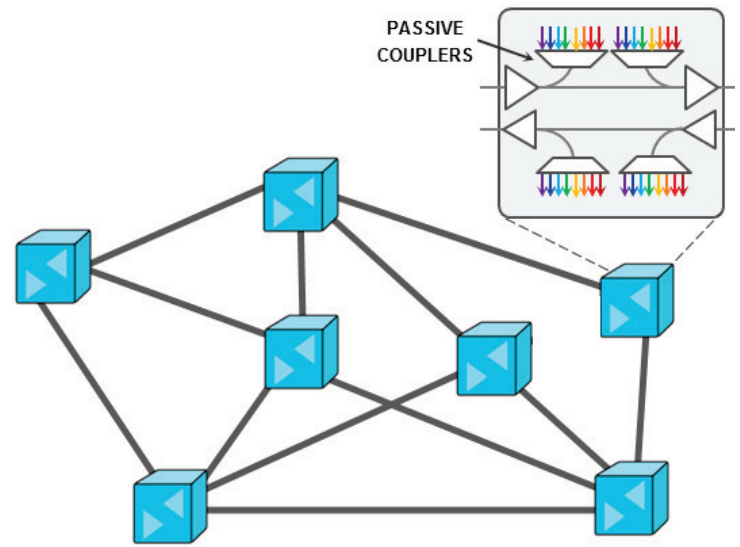

Filterless architecture

Fig. 1 Contrasting active photonic switching networks and filterless networks.

Protocol (IP) layer to be handled more cost-effectively at a lower network layer without reconfiguring the intermediate nodes. Simpler impairment-aware routing in filterless networks makes SDN control more straightforward.

\section{Performance Analysis and Design Trade-OfFs FOR FILTERLESS NETWORKS}

A two-step approach has been used for filterless network design [5]. In the first step, a genetic algorithm is applied to construct sets of interconnected optical fiber links (referred to as fiber trees), based on capacity demand and fiber topology. The trees are constructed using passive optical splitters and combiners at network junctions. Fiber tree design is subject to network connectivity, laser loop avoidance and system reach constraints. In the second step, static shortest-path routing over the fiber tree is performed for each connection and wavelength assignment is made using a tabu search metaheuristic for graph coloring with the objective to minimize the number of wavelengths.

Filterless solutions have been proposed for terrestrial and submarine network topologies. The first studies were focused on regional and core network applications [6-10]. Unsurprisingly, comparative cost analyses in terrestrial networks have shown significant savings are achievable in the filterless network solutions due to replacement of switching and filtering elements by simple fiber couplers. As shown in Fig. 2, filterless networks can be deployed at a fraction of the cost of ROADM-based networks. Fiber trees offer a number of alternate paths for protection but additional paths likely need to be constructed to ensure $1+1$ protection for all wavelength or spectrum channels. Filterless solutions have been proposed recently for real-world application by a network operator using evolutionary and deterministic algorithms [11].

Similar studies carried out on submarine networks have shown that the filterless technology can reduce the terminal cost by $30-44 \%$ and the line equipment cost by $11-12 \%$ when compared to conventional submarine networks where ROADMs can be deployed only at the cable landing stations [12-15].
Wavelength or spectrum consumption in filterless networks has been studied extensively [6-10,12-14]. Typical performance results are illustrated in Fig. 3. Depending on the physical topology and traffic, fixed-grid filterless network solutions can consume $10-50 \%$ more wavelengths or spectrum than their ROADM-based counterparts.

Filterless optical networks suffer from wavelength or spectrum channel propagation along all branches in the fiber tree beyond the destination node. The presence of these unfiltered signals magnifies wavelength or spectrum consumption, as the spectral resources occupied by these channels cannot be reused for other connections. The addition of wavelength blockers (or colored fixed passive filters) in algorithmically determined locations between fiber trees reduces unfiltered channel propagation and provides extra capacity and connectivity alternatives between nodes. These hybrid solutions are referred to as semi-filterless networks [8].

The results show that filterless solutions are good for networks with small numbers of nodes $(\leq 10-12)$ and size (with respect to system transmission reach), as well as good connectivity $(\geq 0.8)$ and high average nodal degree $(\geq 3.0)$. Additional $20-30 \%$ savings in spectrum consumption are possible through flex-grid operation which can be achieved at minimal upgrade cost in filterless networks $[9,10]$.

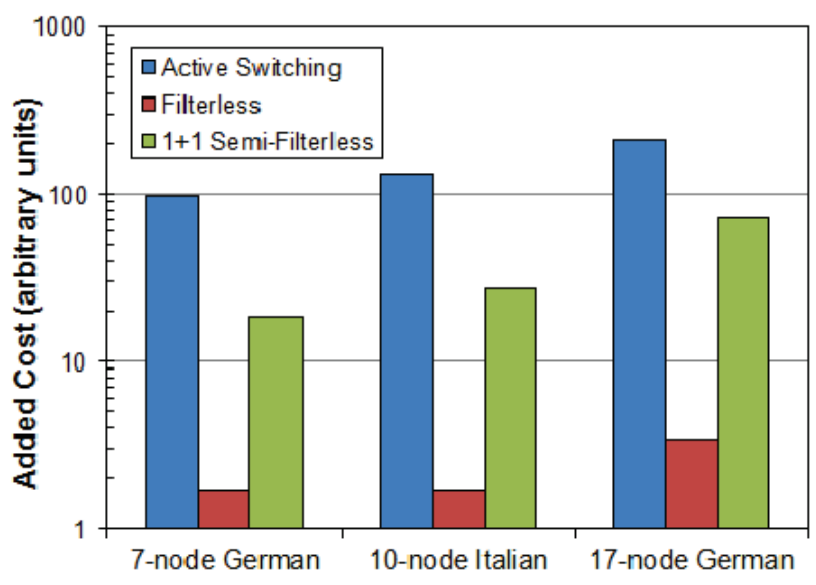

Fig. 2 Comparative cost analysis of conventional and filterless network solutions [8]. 


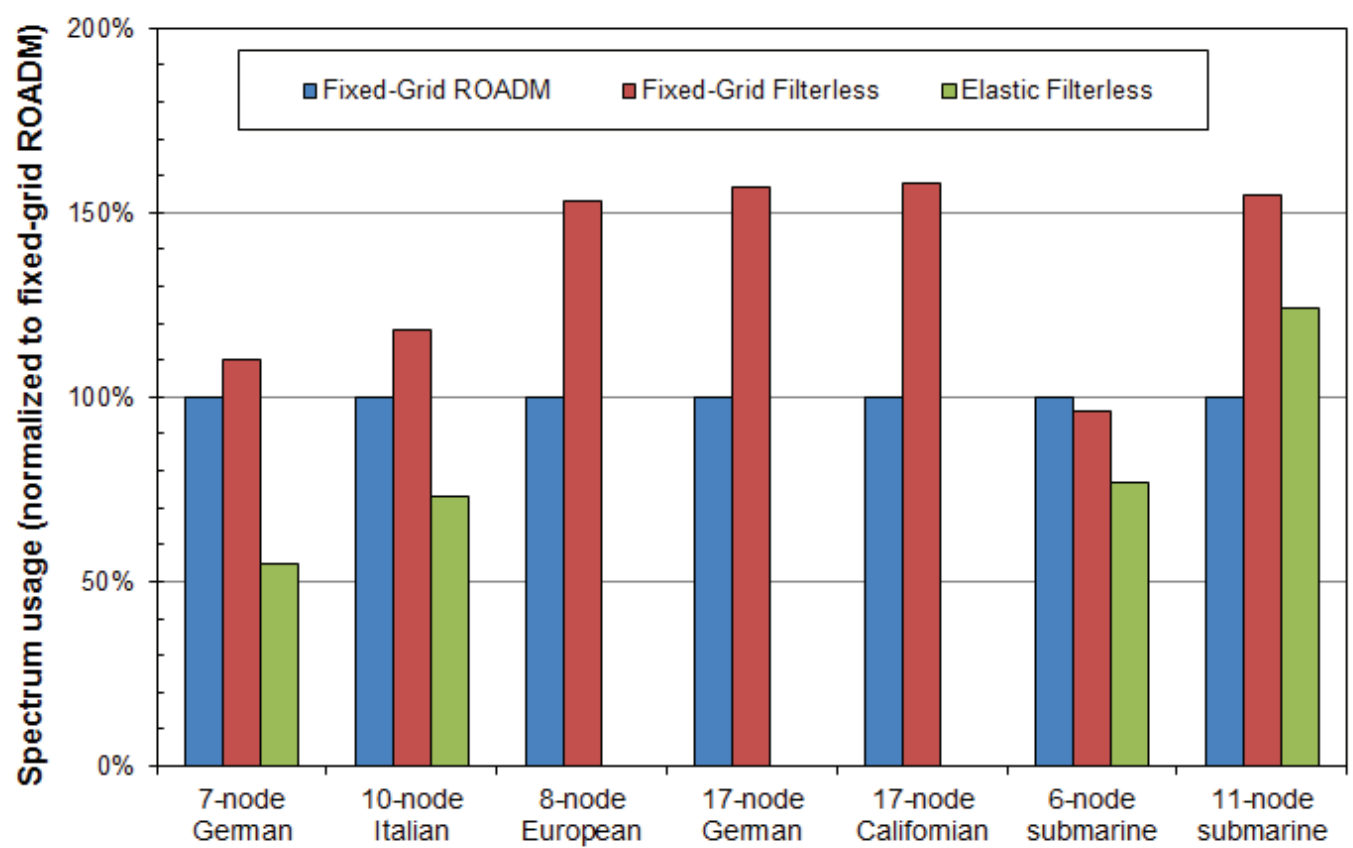

Fig. 3 Wavelength consumption (in relative units) for fixed-grid ROADM, fixed-grid filterless and elastic filterless network solutions. Elastic filterless case: results at $16 \mathrm{~Tb} / \mathrm{s}$ of traffic $[6,10,13,14]$.

A programmable filterless network architecture based on optical white boxes (or spatial switches) has also been proposed to reduce the spectrum consumption in filterless networks at a lower cost than ROADM-based approaches [16].

Finally, a dynamic network resource allocation scheme and algorithm have been proposed to leverage the agility of a filterless multi-span submarine network experiencing daily non-synchronous traffic variations between nodal pairs. The results show that filterless networks can enable significant transceiver and spectrum savings in long haul networks with time-varying traffic load [15].

\section{Challenges with Filterless Optical Networks}

The study of filterless networks has shown that they can definitely offer cost-effective solutions. At high utilization rates they do exhibit capacity limitations compared to active photonic switching solutions, but these limitations can be compensated at least partially through flex-grid operation or the employment of wavelength blocking techniques.

Some other aspects can be considered as potential issues with filterless networks. First, a global network view is needed to understand blocking from unfiltered channels. This problem is conveniently solved by using an external (SDN/PCE) control scheme [17]. The removal of per channel power adjustment in WSS can cause optical power management problems and potentially limit fiber tree size and optical reach. This power balancing problem can be mitigated by adjusting individual channel launch power levels. Some other physical aspects need to be managed in filterless networks. Optical loops must be avoided in creating the fiber trees to prevent laser effects. Noise funneling due to the absence of filtering needs to be taken into account in determining receiver penalty $[18,19]$.

Security can also be considered as a concern in filterless networks due to its broadcast nature making channels available to receivers beyond their destinations. These concerns can be addressed by using data encryption with deciphering key exchange. Digital signal processing (DSP)-assisted modems also provide an additional barrier against eavesdropping through dynamic distribution of propagation impairment compensation between transceiver pairs making recovery difficult at geographically disparate sites.

\section{CONCLUSION}

Table 1 summarizes the main findings of these studies between active and filterless networks according to different performance criteria (from cost to agility). The results show that filterless network solutions perform relatively well in metropolitan, regional, and core application areas when the demand is relatively low compared to the network capacity. In core mesh networks, filterless networks cost significantly less than ROADM-based networks and their functionalities are equivalent. However, their capacity is constrained compared to active photonic switching networks.

The economics of submarine filterless networks are also promising; however, further practical evaluation is needed before conclusions can be drawn on the applicability of this approach in undersea applications.

Based on the cost and spectrum consumption results, we can conclude that filterless networks can be considered as a valuable network technology in metropolitan and aggregation networks. 


\begin{tabular}{|c|c|c|c|c|c|c|c|c|c|c|c|c|}
\hline \multirow{3}{*}{$\begin{array}{l}\text { Characteristic } \\
\begin{array}{l}\text { Demand relative } \\
\text { to network capacity }\end{array}\end{array}$} & \multicolumn{4}{|c|}{ Metro - Regional - Core } & \multicolumn{4}{|c|}{ Submarine } & \multicolumn{4}{|c|}{ Backhaul - Distribution } \\
\hline & \multicolumn{2}{|c|}{ Active } & \multicolumn{2}{|c|}{ Filterless } & \multicolumn{2}{|c|}{ Active } & \multicolumn{2}{|c|}{ Filterless } & \multicolumn{2}{|c|}{ Active } & \multicolumn{2}{|c|}{ Filterless } \\
\hline & Low & High & Low & High & Low & High & Low & High & Low & High & Low & High \\
\hline Agility & & & & & & & & & & & & \\
\hline Reliability & & & & & & & & & & & & \\
\hline Operations & & & & & & & & & & & & \\
\hline Spectrum consumptic & & & & & 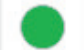 & 0 & C & & & & & \\
\hline Solution complexity & & & & & & & & & & & & \\
\hline
\end{tabular}

Table 1 Suitability of filterless and active photonic switching solutions in different network applications. Legend: green $=$ good, white $=$ acceptable, yellow $=$ poor, red $=$ unacceptable .

\section{ACKNOWLEDGMENTS}

This work was supported by the Natural Sciences and Engineering Research Council (NSERC) of Canada under grants CRDPJ 335465-05 and CRDPJ 435462-12, as well as by Nortel Networks and Ciena. The authors acknowledge the contribution of Émile Archambault, Guillaume Mantelet, Zhenyu Xu, Marija Furdek, Md. Nooruzzaman and Nabih Alloune to network design algorithms.

\section{REFERENCES}

[1] C. Tremblay, F. Gagnon, B. Châtelain, É. Bernier, M. P. Bélanger, "Filterless Optical Networks: A Unique and Novel Passive WAN Network Solution," $12^{\text {th }}$ Optoelectron. Commun. Conf./16 ${ }^{\text {th }}$ Int. Conf. Integrated Opt. Opt. Fiber Commun. (OECC/IOOC 2007), Yokohama, Japan, July 9-13, 2007, Paper 12P-7.

[2] A. Clauberg, "IPv6 deployment in Germany and Croatia," [Online]. Available:

http://www.ipv6observatory.eu/wp-content/uploads/2012/11/01-06-Axel -Clauber1.pdf.

[3] Filterless networks, IEEE TAOS Newsletter, no. 3, June 2015.

[4] M. Gunkel, A. Mattheus, F. Wissel, A. Napoli, J. Pedro, N. Costa, T. Rahman, G. Meloni, F. Fresi F. Cugini. N. Sambo and M. Bohn, "Vendor-interoperable elastic optical interfaces: Standards, experiments, and challenges," IEEE/OSA J. Opt. Commun. Netw., vol. 7, no. 12, pp. B184-B193, December 2015.

[5] É. Archambault, D. O'Brien, C. Tremblay, F. Gagnon, M. P. Bélanger, E. Bernier, "Design and Simulation of Filterless Optical Networks: Problem Definition and Performance Evaluation," IEEE/OSA J. Opt. Commun. Netw., vol. 2, no. 8, pp. 496-501, 2010.

[6] C. Tremblay, É. Archambault, M. P. Bélanger, F. Gagnon, D. V. Plant, "Passive filterless core networks based on advanced modulation and electrical compensation technologies," Telecommun. Syst., vol. 54, no. 2, pp. 167-181, July 2013.

[7] G. Mantelet, A. Cassidy, C. Tremblay, D. V. Plant, P. Littlewood, M. P. Bélanger, "Establishment of dynamic lightpaths in filterless optical networks," IEEE/OSA J. Opt. Commun. Netw., vol. 5, no. 9, pp. 1057-1065, September 2013.

[8] Z. Xu, É. Archambault, C. Tremblay, J. Chen, L. Wosinska, M. P. Bélanger, P. Littlewood, "1+1 Dedicated Optical-Layer Protection Strategy for Filterless Optical Networks," IEEE Commun. Lett., vol. 18, no. 1, pp. 98-101, January 2014.

[9] Z. Xu, C. Tremblay, É. Archambault, M. Furdek, J. Chen, L. Wosinska, P. Littlewood, M. P. Bélanger, "Flexible Bandwidth Assignment in
Filterless Optical Networks," IEEE Commun. Lett., vol. 19, no. 4, pp. 565-568, April 2015

[10] É. Archambault, N. Alloune, M. Furdek, Z. Xu, C. Tremblay, A. Muhammad, J. Chen, L. Wosinska, P. Littlewood, M. P. Bélanger, "Routing and Spectrum Assignment in Elastic Filterless Optical Networks," IEEE/ACM Trans. Netw., vol. 24, no. 6, pp. 3578-3592, December 2016.

[11] S. Krannig, R. Moegel, M. Gunkel, T. Kussel, N. Michaelis, F. Wissel, B. Drossel, "How to design an optimized set of fibre-trees for filterless optical networks - The elegance of a multi-goal evolutionary Pareto optimization versus a deterministic approach," ITG-Symposium: Photonic Networks 2016, Leipzig, Germany, May 12-13, 2016, pp. 51-58.

[12] M. Nooruzzaman, N. Alloune, F. Nabet, Z. Xu, É. Archambault, C. Tremblay, M. Furdek, J. Chen, L. Wosinska, P. Littlewood, M. P. Belanger, "Flexible Architecture for Coherent Undersea Networks," Optical Network Design and Modeling (ONDM) 2015, Pisa, Italy, May 11-14, 2015, 68-73.

[13] M. Nooruzzaman, F. Nabet, N. Alloune, É. Archambault, C. Tremblay, M. Furdek, J. Chen, L. Wosinska, P. Littlewood, M. P. Bélanger, "Resource Savings in Gridless Coherent Submarine Networks with Filterless Architectures," SubOptic 2016, Dubai, April 18-21, 2016, Paper NA08.

[14] M. Nooruzzaman, N. Alloune, F. Nabet, É. Archambault, C. Tremblay, M. Furdek, J. Chen, L. Wosinska, P. Littlewood, M. P. Bélanger, "Agile Filterless Submarine Ring Networks," $17^{\text {th }}$ Int. Netw. Strategy Planning Symposium (NETWORKS 2016), Montreal, Canada, September 26-28, 2016, Paper 409.

[15] M. Nooruzzaman, N. Alloune, C. Tremblay, P. Littlewood, M. P. Bélanger, "Resource Savings in Submarine Networks Using Agility of Filterless Architectures", IEEE Commun. Lett., vol. 21, no. 3, pp. 512-515, March 2017.

[16] M. Furdek, A. Muhammad, G. Zervas, N. Alloune, C. Tremblay, L. Wosinska, "Programmable Filterless Network Architecture Based on Optical White Boxes," Optical Network Design and Modeling (ONDM) 2016, Cartagena, Spain, May 9-12, 2016.

[17] G. Mantelet, C. Tremblay, D. V. Plant, P. Littlewood, M. P. Bélanger, "PCE-based Centralized Control Plane for Filterless Networks," IEEE Commun. Mag., vol. 51, no. 5, pp.128-135, May 2013.

[18] J.-P. Savoie, C. Tremblay, D. V. Plant, M. P. Bélanger, "Physical Layer Validation of Filterless Optical Networks," $36^{\text {th }}$ European Conf. Opt. Commun. (ECOC'10), Sept. 19-23, 2010, Torino, Italy, Paper P5.08.

[19] A. Cassidy, C. Tremblay, M. P. Bélanger, P. Littlewood, "Dynamic Performance of Filterless Optical Networks: Network Resources and Transmission Quality," 14 ${ }^{\text {th }}$ SPIE Photonics North Conf., Montreal, Canada, June 6-8, 2012, Paper COMM-6-P-4. 